\title{
Surfactant TWEEN20 provides stabilisation effect on anthocyanins extracted from red grape pomace
}

Article

Accepted Version

Creative Commons: Attribution-Noncommercial-No Derivative Works 4.0

MohdMaidin, N., Oruna-Concha, M. J. and Jauregi, P. (2019) Surfactant TWEEN20 provides stabilisation effect on anthocyanins extracted from red grape pomace. Food Chemistry, 271. pp. 224-231. ISSN 0308-8146 doi: https://doi.org/10.1016/j.foodchem.2018.07.083 Available at https://centaur.reading.ac.uk/79337/

It is advisable to refer to the publisher's version if you intend to cite from the work. See Guidance on citing.

Published version at: http://dx.doi.org/10.1016/j.foodchem.2018.07.083

To link to this article DOI: http://dx.doi.org/10.1016/j.foodchem.2018.07.083

Publisher: Elsevier

All outputs in CentAUR are protected by Intellectual Property Rights law, including copyright law. Copyright and IPR is retained by the creators or other copyright holders. Terms and conditions for use of this material are defined in the End User Agreement.

www.reading.ac.uk/centaur 
Central Archive at the University of Reading

Reading's research outputs online 
1 Surfactant TWEEN20 provides stabilisation effect on anthocyanins extracted 2 from red grape pomace

${ }^{\text {a }}$ Department of Food and Nutritional Sciences, Harry Nursten Building, University of Reading, RG6 6AP, Reading, United Kingdom

${ }^{b}$ Department of Food Technology, Faculty of Food Science and Technology, University *corresponding author: Paula Jauregi; Email address: p.jauregi@ reading.ac.uk

10 Present address: Department of Food and Nutritional Sciences, Harry Nursten Building,

University of Reading, RG6 6AP, Reading, United Kingdom

\section{Abstract}

14 Red grape pomace, a wine-making by-product is rich in anthocyanins and has many applications in food and pharmaceutical industry. However, anthocyanins are unstable during processing and storage. This study aimed to investigate the stability of anthocyanins

17 obtained by hydroalcoholic extraction (with and without sorbic acid) and colloidal gas

18 aphrons (CGA) separation; a surfactant (TWEEN20) based separation. Anthocyanins in 19 CGA samples showed higher stability (half-life $=55 \mathrm{~d}$ ) than in the crude extract (half-life=

$2043 \mathrm{~d}$ ) and their stability increased with the concentration of TWEEN20 in the CGA fraction 21 (6.07-8.58mM). The anthocyanins loss in the CGA sample (with the maximum content of 22 surfactant, $8.58 \mathrm{mM}$ ) was $34.90 \%$, comparable to that in the crude ethanolic extract with 23 sorbic acid (EE-SA) (31.53\%) and lower than in the crude extract (44\%). Colour stabilisation 24 was also observed which correlated well with the stability of individual anthocyanins in the 25 EE and CGA samples. Malvidin-3-o-glucoside was the most stable anthocyanin over time.

27 Keywords: Grape pomace, anthocyanins stability, colloidal gas aphrons, surfactant, storage 
Abbreviations: ABTS, 2,2'-azino-bis(3-ethylbenzothiazoline-6-sulphonic acid); AOP, antioxidant power; CGA, colloidal has aphrons; EE; ethanolic extract, EE-SA, ethanolic extract with addition of sorbic acid; GAE, gallic acid equivalents; $\mathrm{t}_{1 / 2}$, half-life; glc, 3-oglucoside; ME, malvidin glucoside equivalent; V4, CGA fraction separated at volumetric ratio 4; V8, CGA fraction separated at volumetric ratio 8; V16, CGA fraction separated at 33 volumetric ratio 16.

\section{Introduction}

Grapes are one of the most important fruit crop cultivated across the world, whereby $80 \%$ of the grape productions are used in wine-making industry (Fontana, Antoniolli, \& Bottini, 2013). Wine production is considered one of the most important agricultural activities, generating large amount of residues including grape skins, stems and seeds (Yu \&

40 Ahmedna, 2013). At the end of the fermentation process, large amounts of resiudes are being

41 discharged containing high amount of phenolic compounds including anthocyanins,

42 catechins, flavonol glycosides, phenolic acids and stilbenes (Kammerer, Kammerer, Valet,

$43 \&$ Carle, 2014). This is seen by the environmental management authorities as a serious threat

44 because they are low in $\mathrm{pH}$ and high in organic matter thus potentially causing a phytotoxic

45 effect if applied to crops or wetlands (Kammerer, Claus, Carle, \& Schieber, 2004; Lavelli,

46 Harsha, Laureati, \& Pagliarini, 2017). Therefore, converting and utilising this by-product to

47 another useful product would be a solution to this problem. For instance, the anthocyanins

48 from this pomace can be used as natural food colourant (Thakur \& Arya, 1989).

49 Anthocyanins are sensitive to thermal degradation making the recovery rather difficult and

50 complex, but they are on demand due to their wide applications in food (already being used

51 as food colourants, E163, approved by EC) as well as in pharmaceuticals and cosmetics.

52 Thus, various extraction techniques have been studied and used, including acidified alcohol,

53 sub- and supercritical fluid and high pressure processing (Barba, Zhu, Koubaa, Sant'Ana, \&

54 Orlien, 2016; Drosou, Kyriakopoulou, Bimpilas, Tsimogiannis, \& Krokida, 2015;

55 Lozovskaya, Brenner Weiss, Franzreb, \& Nusser, 2012). 
Food processing generally involves thermal processing prior to consumption and this

57 process has a great influence on the anthocyanins content in the final product. Thermal 58 processing involves high temperatures ranging from $50^{\circ} \mathrm{C}$ to $150^{\circ} \mathrm{C}$, depending on the $\mathrm{pH}$ 59 and the desired shelf life of the product. Anthocyanins are expected to degrade over time.

60 However, the storage temperature has been found to be an important factor that is affecting 61 anthocyanins' shelf life. Degradation of anthocyanins is greatly affected by the type of 62 anthocyanin, the origin of the samples and the storage temperature (Hellström, Mattila, \& 63 Karjalainen, 2013). The thermal degradation of anthocyanins in extracts and model systems 64 are reported to follow first-order reaction kinetics (Presilski, Presilska, \& Tomovska, 2016). The stability of anthocyanins can be improved, by self-association of the anthocyanins, removal of oxygen and inactivation of enzymes (Hellström et al., 2013). In the food

67 industry, the sensitivity of bioactive compounds is addressed by incorporating edible 68 coatings as a structural matrix, used widely to create a barrier from oxygen, moisture and 69 solute movement (Falguera, Quintero, Jiménez, Muñoz, \& Ibarz, 2011). Encapsulating methods such as spray drying/spray chilling or liposomes have been used. The former requires liquid droplets or small particles being incorporated within a continuous edible 72 coating, thus it requires an emulsifier. Liposomes are microscopic spherical particles 73 consisting of one or more lipid bilayers that can encapsulate or bind a variety of molecules.

74 Therefore, particularly in food applications, food grade surfactants such as TWEEN20 have been used as emulsifying agents to fit this purpose (Quirós-Sauceda, Ayala-Zavala, Olivas, \& González-Aguilar, 2014). Moreover, TWEEN20 has been seen as having a profound protective effect on five different polyphenols, by slowing down the auto-oxidation process at pH 4.5 (Lin, Wang, Qin, \& Bergenståhl, 2007). 
81 different feedstock such as astaxanthin (Dermiki, Bourquin, \& Jauregi, 2010; Dermiki,

82 Gordon, \& Jauregi, 2009), proteins (Fuda \& Jauregi, 2006; Fuda, Bhatia, Pyle, \& Jauregi,

83 2005) and polyphenols (MohdMaidin, Michael, Oruna-Concha, \& Jauregi, 2017; Spigno,

84 Dermiki, Pastori, Casanova, \& Jauregi, 2010; Spigno, Amendola, Dahmoune, \& Jauregi,

85 2015). The type of surfactant (i.e cationic, anionic and non-ionic) determines the outer

86 charge of the CGA, where molecules with the opposite charge will attract to the CGA

87 resulting in their effective separation into the CGA phase.

88 In our previous work it was shown that $70 \%$ of the anthocyanins could be recovered

89 from the ethanolic extract of grape pomace using CGA generated from TWEEN20. The

90 CGA fraction will be rich in surfactant therefore, it will be interesting to test what will be

91 the added value of extracting the anthocyanins in such a solution and whether this can offer

92 any advantage to their formulation for subsequent applications. Thus the present study aimed

93 at assessing the stability of anthocyanins in the CGA separated fraction over time in

94 comparison with their stability in the crude ethanolic extract (EE) (before the CGA

95 separation) as well as in the crude ethanolic extract with a commercial additive, sorbic acid

96 (EE-SA). It is therefore hypothesised that the anthocyanins in the CGA sample will show

97 higher stability than in the crude extract over time.

99 2. Materials and methods

$100 \quad 2.1 \quad$ Materials

101 Grape pomace (Barbera variety) was obtained from a winery in Northern Italy. All the

102 solvents (purity of 95\% and above) used in this project were obtained from Sigma-Aldrich

103 Company Ltd., Dorset, UK. For the HPLC analysis, the solvents used were of HPLC grade 104 (purity of 98-99.9\%) also from Sigma Aldrich. 
The grape pomace (Barbera variety) was kindly provided by a winery located in

107 Nothern Italy. At the winery, the fermented pomace was recovered and oven dried at $60^{\circ} \mathrm{C}$

108 until the residual moisture content is $<5 \%$. The dried pomace powder was sieved with a $5 \mathrm{~mm}$

109 sieve to separate the skins from the seeds and milled into fine powders with particles size <

$1102 \mathrm{~mm}$ and stored in the freezer at $-20^{\circ} \mathrm{C}$ until further use.

111 The extraction procedure was done in accordance to our previous study using ethanol112 aqueous solvent (MohdMaidin et al., 2017). The extract was filtered and two different 113 samples were produced: (1) approximately, $400 \mathrm{~mL}$ of the ethanol extract labeled as EE and

114 (2) another 400mL ethanol extract with sorbic acid (>99\%) (Sigma, UK) and labelled as EE-

115 SA. Sorbic acid was chosen in this study for its wide application as food additive, thus 116 making it closer to the formulation of most low $\mathrm{pH}$ food products and neutral taste (Troller 117 \& Olsen, 1967). Both EE and EE-SA were considered control samples. The remaining 118 filtrate of $800 \mathrm{~mL}$ was kept aside for CGA separation.

\section{$120 \quad 2.3 \quad$ CGA separation using 10mM TWEEN20}

The separation of polyphenols from the crude ethanolic extract was carried out at 122 different volume ratios of CGA to feed $\left(\mathrm{V}_{\mathrm{CGA}} / \mathrm{V}_{\text {feed }}\right)$. The ratios selected were 4,8 and 16 .

123 The separations were individually carried out in a flotation glass column according to the 124 method described in our previous work (MohdMaidin et al., 2017); each separation was 125 carried out in triplicate. It should be noted that as the volumetric ratio increased, so did the 126 concentration of TWEEN20 in the solution of the separated CGA fraction. The concentration 127 of TWEEN20 in each of these fractions was estimated from a knowledge of the separated 128 volume of CGA and corresponding liquid fraction which was determined from a 129 measurement of gas hold-up (gas volumetric ratio defined as the volume of air incorporated 
130 in a given volume of CGA dispersion) of the CGA generated with this solution of TWEEN20

131 (61.3\%). The estimated concentrations were: in $\mathrm{V} 4,6.07 \mathrm{mM}$, in $\mathrm{V} 8,7.56 \mathrm{mM}$ and in $\mathrm{V} 16$,

$1328.58 \mathrm{mM}$. The summary of the extraction and separation process is briefly described in Figure

1331.

134

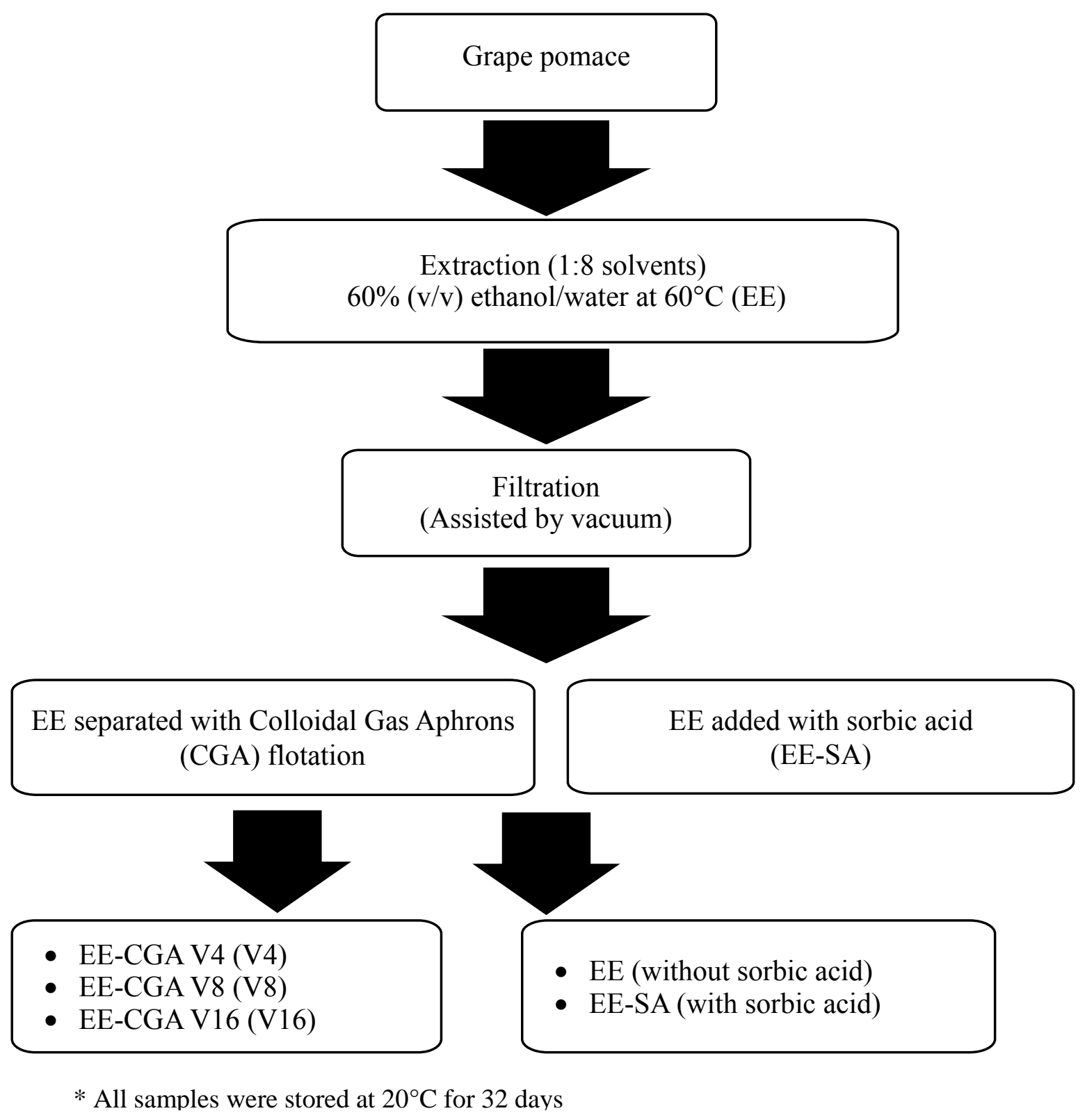

Figure 1: Flow diagram of hydroalcoholic extraction and CGA separation processes applied

137 to grape pomace, $\mathrm{n}=3$; EE is the ethanolic extract; EE-SA is the ethanolic extract with 138 addition of sorbic acid; EE-CGA V4 is the ethanolic extract further processed with CGA at 139 CGA to feed volumetric ratio of 4; V8 and V16 correspond to the extracts further processed 140 with CGA at CGA to feed volumetric ratios 8 and 16 respectively 
142 Briefly, the EE, EE-SA and CGA fractions were divided in equal volumes and kept in

143 sterilised containers in the darkness. These were then stored at room temperature $20^{\circ} \mathrm{C}$ (SD

$1441^{\circ} \mathrm{C}$ ) which was regularly monitored using a thermometer for 32 days. The total phenolic

145 content, total anthocyanin and antioxidant activity were determined as described in section

146 2.5-2.7. The total anthocyanins, individual anthocyanins, antioxidant capacity and the colour

147 degradation over time (32 days; every day for the first 7 days and subsequently 5 days 148 intervals) were determined.

149 The kinetics of degradation of total anthocyanins and individual anthocyanins were 150 assessed; the natural logarithms of these were plotted against time in order to test for first151 order kinetics as described by the equation below:

$$
-\ln \left(\frac{A_{t}}{A_{0}}\right)=k * t
$$

155 Where $\mathrm{A}_{0}$ is the initial anthocyanin content, $\mathrm{A}$ is the anthocyanin content at time $t, t$ is the 156 storage time and $k$ is the rate constant. The degradation rate constant $(k)$ was determined 157 from the slope of the straight line obtained when plotting $\operatorname{Ln}\left(\mathrm{At} / \mathrm{A}_{0}\right)$ vs $t$. From the equation 158 above, the time taken for the anthocyanin content to halve, the half-life $\left(\mathrm{t}_{1 / 2}\right)$, can be derived 159 as:

160

161

$$
t \frac{1}{2}=\frac{\operatorname{Ln}(2)}{k}
$$


164 Folin Ciocalteu (FC) colorimetry method (Singleton \& Rossi, 1965) was employed to 165 determine the total phenolic content of the EE and EE-SA (control samples) and also in all 166 of the CGA processed samples. This method involves the oxidation of phenols using a 167 molybdotungstate reagent to yield a coloured product which can be measured at 760nm using 168 a spectrophotometer (Biotech Ultrospec 1100 pro UV spectrophotometer). Gallic acid 169 (Sigma-Aldrich, UK) standards with concentrations ranging from $0-1000 \mathrm{mg} / \mathrm{L}$ were used to 170 generate standard plots $\left(\mathrm{R}^{2}=0.9881\right)$ and an equation for the calculation of the total phenolic 171 concentration in each extract. The analysis was done in triplicate. The total phenolic content 172 in the CGA processed samples were compared to the controlled samples over time.

\section{$174 \quad 2.6 \quad$ Evaluation of in vitro antioxidant activity}

175 The antioxidant activity of the control samples (EE and EE-SA) along with the CGA 176 processed samples were evaluated according to Re et al., (1999) by the ABTS assay. This 177 method assesses the ability of the antioxidants to scavenge the radical (ABTS) which was 178 determined by measuring the decrease in its absorbance at $734 \mathrm{~nm}$ using a spectrophotometer 179 (Biotech Ultrospec 1100 pro UV spectrophotometer). Different concentrations (0-2000 $\mu \mathrm{M})$ 180 of Trolox standard were used to construct a calibration curve $\left(\mathrm{R}^{2}=0.9991\right)$. The analysis was 181 done in triplicate. The antioxidant activity of the CGA processed samples was compared 182 against that of the control samples, expressed as $\mu \mathrm{M}$ Trolox equivalent. The ratio of 183 percentage inhibition to the total phenolic content of all samples, termed as specific 184 antioxidant power (AOP), was calculated. 


\subsection{Total anthocyanins content}

The total monomeric anthocyanins of control samples, EE and EE-SA along with the

188 CGA processed samples were determined over time using the $\mathrm{pH}$ differential method 189 approved by AOAC (Lee, Rennaker, \& Wrolstad, 2008). This method is based on the

190 anthocyanins structural transformation that occurs with a change in $\mathrm{pH}$. Briefly, the extract 191 was mixed individually with $\mathrm{pH} 1.0$ and 4.5 buffer solutions in a ratio of 1:5 and left for 20 192 minutes. The absorbance of the test portions at both $\mathrm{pHs}$ were determined 193 spectrophotometrically (Biotech Ultrospec 1100 pro UV spectrophotometer) at a wavelength 194 of 520nm and 700nm. The results of the anthocyanin pigment were expressed as malvidin195 3-glucoside equivalents (ME) according to equation 3.

Where $\mathrm{A}=\left(\mathrm{A}_{520 \mathrm{~nm}}-\mathrm{A}_{700 \mathrm{~nm}}\right)_{\mathrm{pH} 1.0}-\left(\mathrm{A}_{520 \mathrm{~nm}}-\mathrm{A}_{700 \mathrm{~nm}}\right)_{\mathrm{pH} 4.5} ; \mathrm{MW}($ molecular weight of malvidin-

3 -glucoside $=493.43 \mathrm{~g} / \mathrm{mol} ; \mathrm{DF}=$ dilution factor; $1=$ path length in $\mathrm{cm} ; \varepsilon=28000$ molar extinction coefficient and $10^{3}=$ factor for conversion from $\mathrm{g}$ to $\mathrm{mg}$ and $\mathrm{cm}$.

\subsection{Identification and Quantification of Anthocyanins by HPLC}

The separation of the polyphenols was performed using an Agilent HPLC 1100 series equipped with a degasser, a quaternary pump and a photodiode array detector model

206 (Agilent, Waldbronn, Germany) with Chemstation software. The column used was a C18

207 HiChrom (150 mm x $4.6 \mathrm{~mm}$ i.d; $5 \mu \mathrm{m}$ particle size and $100 \AA$ A pore size; part no.EXL-121-

$2081546 \mathrm{U}$ ) operated at $30^{\circ} \mathrm{C}$. The separation method was the same as described in our previous paper (MohdMaidin et al., 2017). The polyphenols were monitored at 280nm and the UV/Vis 
210 spectra were recorded in the range of 200 to $760 \mathrm{~nm}$. The main anthocyanins were detected

211 at 520nm and identified based on the retention times and by comparing the spectra with that

212 of the external standards which were: delphinidin-3-o-glucoside (>99\%) $(\mathrm{RT}=4.8$;

213 calibration curve $\left.\mathrm{R}^{2}=0.8771\right)$; cyanidin-3-o-glucoside $(>98 \%)(\mathrm{RT}=7.8$; calibration curve

$\left.214 \mathrm{R}^{2}=0.98744\right)$; petunidin-3-o-glucoside $(>98 \%)\left(\mathrm{RT}=8.5\right.$; calibration curve $\left.\mathrm{R}^{2}=0.99702\right)$ and

215 malvidin-3-o-glucoside ( $>99 \%)\left(\mathrm{RT}=7.8\right.$; calibration curve $\left.\mathrm{R}^{2}=0.99994\right)$; all supplied by

216 Extrasynthese, Paris, France.

2182.9 Determination of CIELab colour parameters and $\mathrm{pH}$

219 The changes in colour of the EE, EE-SA and the CGA processed samples were 220 measured using a CT-1100 ColourQuest HunterLab by taking the measurements in 221 transmittance mode. Standard black plates were used for standardization. $\mathrm{L}^{*}, \mathrm{a}^{*}$ and $\mathrm{b}^{*}$ 222 measurements were obtained and used to calculate chroma and hue angles based on 223 equations 4 and 5 below. Delta $\mathrm{E}(\Delta \mathrm{E})$ was calculated based on the changes of the values of $224 \mathrm{~L}^{*}, \mathrm{a}^{*}$ and $\mathrm{b}^{*}$ at a given time, in comparison to these values at day 0 and applying equation 2256.

$$
\text { Hue angle }\left(^{\circ}\right)=\left[180 *\left(\frac{\operatorname{ATAN}\left(\frac{b}{a}\right)}{\pi}\right)\right]+\left(\frac{b}{a}\right) \quad(\text { Equation } 4)
$$

227 Chroma $=\sqrt{a^{2}+b^{2}} \quad($ Equation 5)

$$
\Delta E=\left[(\Delta L)^{2}+(\Delta a)^{2}+(\Delta b)^{2}\right]^{\frac{1}{2}} \quad \text { (Equations 6) }
$$


231 diagram to identify colour and monitor changes. The changes of colour in all the CGA

232 processed samples over time were compared to EE and EE-SA as the control samples.

233 The $\mathrm{pH}$ of all samples was monitored regularly with a $\mathrm{pH}$ meter (Mettier-Toledo

234 SevenEasy), which was calibrated by using pH 4.0 and 7.0 buffer solutions (Sigma, UK).

\section{$236 \quad 2.10 \quad$ Statistical analysis}

All the experiments were performed in triplicate. The data were subjected to the analysis of variance using IBM® SPSS $®$ Statistics 21 software program where statistical

239 differences were noted. Differences among the different treatments were determined by

240 using the Tukey test. The significance level was defined at $p<0.05$. The results were reported 241 as means $\pm \mathrm{SD}$.

$243 \quad 3 . \quad$ Results and Discussion

244 3.1. Changes of total phenolic content over storage time

245 Initial values for total phenolic content was measured in control samples (EE and EE246 SA) and three of the CGA processed samples at day 0. The total phenolic content for all 247 samples range was 285-2080 mg GAE/L. The TP content for EE-SA was higher $(p=0.0371)$

248 than EE, which can possibly be explained by the presence of sorbic acid. The total 249 monomeric anthocyanin range was 99.1-422.9 $\mathrm{mg} \mathrm{ME} / \mathrm{L}$. The antioxidant activity range was 250 2299-14469 $\mu \mathrm{M}$ Trolox equivalent.

Over a storage period of 32 days, the losses in the TP content were minimal in all the 252 samples (Fig. 2A). The maximum degradation observed in EE-SA and EE was not more than $25310 \%$. Among all the CGA processed samples, the lowest losses of the TP content was in V16 
254 (4.91\%), followed by V8 (5.44\%) and finally V4 (6.42\%), although they were not

255 significantly different $(p=0.062)$.
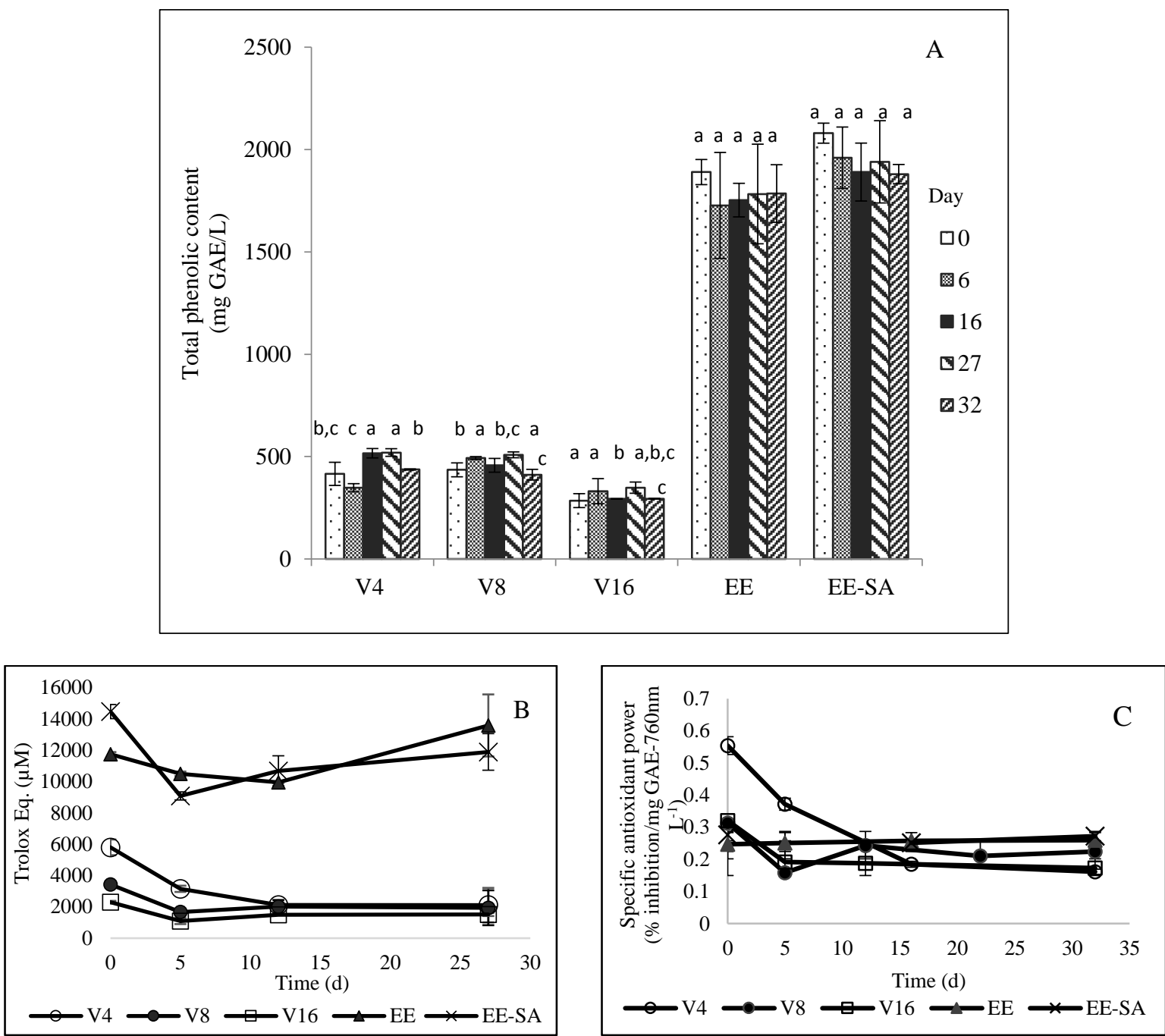

260 Figure 2. Total phenolic content (A), antioxidant activity profile expressed as Trolox Equivalent $261(\mu \mathrm{M})(\mathrm{B})$, and specific antioxidant power (\% inhibition/mg GAE-760nm L-1), (C) of CGA processed 262 and control samples over time. Error bars represent means \pm SD $(n=3)$. V4, CGA fraction separated 263 at volumetric ratio 4; V8, CGA fraction separated at volumetric ratio 8; V16, CGA fraction separated 
264 at volumetric ratio 16; EE, crude ethanolic extract and EE-SA, ethanolic extract with sorbic acid.

265 Different letter within each series indicates significant differences using Tukey's test $(\mathrm{P}<0.05)$.

\subsection{Changes in antioxidant activity over storage time}

269 The antioxidant activity of the control samples and CGA processed samples stored 270 over time was evaluated using ABTS assay. Figure 2 (B) shows a decrease in antioxidant 271 activity in both, the control samples and the CGA processed samples over time. The 272 reduction in antioxidant activity was clearly observed for all samples during the first 5 days.

273 Further decrease was observed in V4 after 5 days, however in V8, V16 and control samples 274 no further reductions or even slight increases in antioxidant activity was observed after this 275 time.

Moreover, when the specific antioxidant power (calculated as percentage of inhibition per total phenol content) was calculated, a more distinct pattern was observed (Fig. 2C). In

278 general, the specific antioxidant power decreased over time at a higher rate than the total 279 phenols (Fig. 2A). Rapid loss of antioxidant power was observed particularly in V4 from 280 day 0 to day 16 , and in V8, V16 and EE-SA only over the first 5 days. It was interesting to 281 note that there was no specific antioxidant loss in EE. This could be related to the total 282 phenolic content as depicted in Figure 2A where the losses in EE were not significantly 283 different $(\mathrm{p} \leq 0.05)$ between the time points. This implied that the losses in TP and antioxidant 284 activity in EE were in the same proportion hence the antioxidant efficiency was almost 285 constant over time (Fig. 2B). However, this was not the case for the CGA samples where 286 minimal losses of total phenolic content were noted but important changes in antioxidant 287 activity were observed. Therefore, this suggests that in these samples the antioxidant activity 288 may not be solely derived from the total phenolic content and/or that the phenolics undergo some chemical changes that affect their antioxidant activity. Over estimation of total 
phenolic content could possibly happen by the high sugar content or ascorbic acid in the

\subsection{Kinetics of total anthocyanins degradation over storage time}

Degradation of anthocyanins has been previously studied in wine and its residues

(Bimpilas, Panagopoulou, Tsimogiannis, \& Oreopoulou, 2016; Clemente \& Galli, 2011,

2013; Lavelli et al., 2017). The patterns of degradation involving anthocyanins are complex,

but the degradation rate generally follows first-order kinetics (Amendola, De Faveri, \& anthocyanin degradation in the presence of surfactant is lacking. In this study, the degradation of anthocyanins in the control samples (EE and EE-SA) were compared with the CGA processed samples stored at $20^{\circ} \mathrm{C}$, which also followed first-order kinetics (Fig. 3).

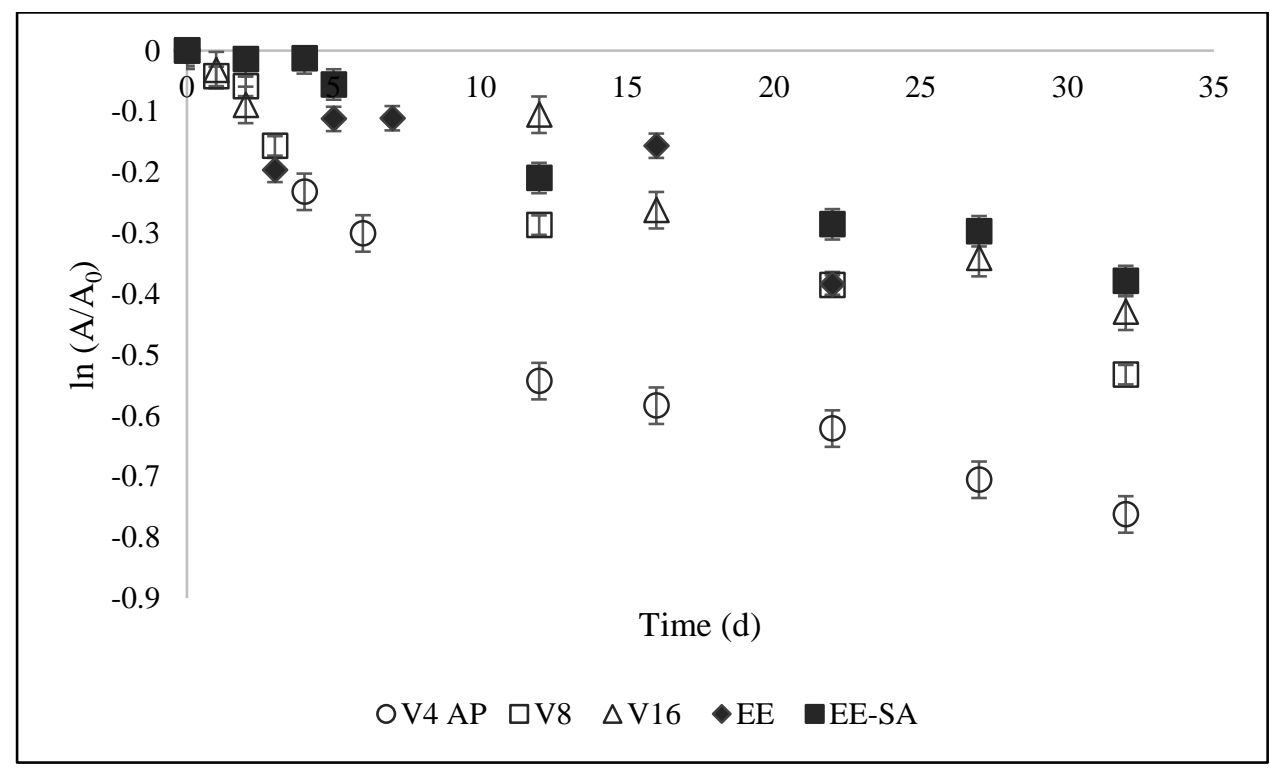

Figure 3. Time course for the decrease of anthocyanins represented here as the natural logarithm of the ratio of anthocyanins concentration at a given time and at time zero $\left(\mathrm{A}_{0}\right)$ during storage at $20^{\circ} \mathrm{C}$. Error bars represent means \pm SD. V4, CGA fraction separated at volumetric ratio 4; V8, CGA fraction separated at volumetric ratio 8; V16, CGA fraction separated at volumetric ratio 16; EE, crude 
309 data in Figure 3. In Table 1A the first-order rate constant $(k)$ and the linear regression

310 coefficient $\left(\mathrm{R}^{2}\right)$ of all samples are shown. The first-order rate constant for anthocyanins

311 degradation ranged between 0.0124 and $0.0217 \mathrm{~d}^{-1}$. Although the $\mathrm{R}^{2}$ values for $\mathrm{V} 4$ and $\mathrm{EE}$

312 were lower than the others, first-order kinetics were assumed. This was also based on the

313 assumption that since the $\mathrm{pH}$ of these samples ranged 3.5-3.8 the degradation of the

314 anthocyanins and thus the ionization of flavillium ion followed first-order kinetics as found

315 by West \& Mauer (2013). The first-order rate constant of EE was higher $\left(k=0.0159 \mathrm{~d}^{-1}\right)$

316 than the one of EE-SA; the first-order rate constant of EE-SA was the lowest among all

317 samples $\left(k=0.0121 \mathrm{~d}^{-1}\right)$. This suggests that the addition of sorbic acid increased the stability

318 of anthocyanins during storage although sorbic acid has only been reported to prevent 319 microbial growth during storage (Troller \& Olsen, 1967).

As shown in Table 1A the first-order rate constant decreased with the increase in volumetric ratio and thus with an increase in surfactant content in the CGA fractions (ranging from 6.07-8.58mM TWEEN20). The samples with the highest stability were the EE-SA and the CGA fraction with the highest surfactant concentration, V16, followed by V8 and EE which had very similar stability. The sample with the lowest concentration of surfactant V4, was found to degrade the fastest over time. proposed to recover phenolic-rich coloured extracts with $36-62 \%$ of total anthocyanins 328 composition (Cardona, Lee And, \& Talcott, 2009). However, the colour degradation of these water-based extracts at $30^{\circ} \mathrm{C}$ is fast, with first-order rate constants of 0.0364 and 0.038 for cold and hot pressed extractions, respectively. In the present study, the first-order rate

331 constants were lower indicating more stable extracts. The most stable sample was the EE332 SA $\left(k=0.0121 \mathrm{~d}^{-1}\right)$ suggesting a stabilisation effect of sorbic acid. Comparable results were 
333 obtained for V16 CGA with $k=0.0124 \mathrm{~d}^{-1}$. However, interestingly the stabilisation effect in

334 the CGA processed samples was only achieved above a certain concentration of surfactant

335 as in V4 and V8 CGA samples the observed stabilisation effect when compared against the

336 EE sample was minimal. It was estimated that the surfactant concentration in V16, V8 and

337 V4 was $8.58 \mathrm{mM}, 7.56 \mathrm{mM}$ and $6.07 \mathrm{mM}$ respectively (see Methods). Therefore the

338 concentration of surfactant in the samples should be at or above $8.58 \mathrm{mM}$ (about 1\%) in order

339 to have a stabilisation effect.

340

Table 1A. First-order empirical rate constants (k) and half-life for anthocyanins.

\begin{tabular}{ccccc}
\hline Sample & $\mathbf{R}^{\mathbf{2}}$ & $\boldsymbol{K}\left(\mathbf{d}^{-\mathbf{1}}\right)$ & $\mathbf{t}_{\mathbf{1} / \mathbf{2}}(\mathbf{d})$ & Loss $(\boldsymbol{\%})^{*}$ \\
\hline V4 & 0.8861 & $0.0217 \pm 0.0019$ & 31 & 53.35 \\
\hline $\mathbf{V 8}$ & 0.9585 & $0.0157 \pm 0.0024$ & 44 & 41.30 \\
\hline V16 & 0.9385 & $0.0124 \pm 0.0015$ & 55 & 34.90 \\
\hline EE & 0.8131 & $0.0159 \pm 0.0012$ & 43 & 41.04 \\
\hline EE-SA & 0.9583 & $0.0121 \pm 0.0011$ & 57 & 31.53
\end{tabular}

Rate constants are expressed as means \pm SD. V4, CGA fraction separated at volumetric ratio 4; V8, CGA fraction separated at volumetric ratio 8; V16, CGA fraction separated at volumetric ratio 16; EE, crude ethanolic extract and EE-SA, ethanolic extract with sorbic acid.

*anthocyanins loss calculated after day 32

Table 1B. Half-lives $\left(\mathrm{t}_{1 / 2}\right.$, day) and degradation rate $\left(k, \mathrm{~d}^{-1}\right)$ of different anthocyanins in control and CGA samples, stored at $20^{\circ} \mathrm{C}$

\begin{tabular}{lccccc}
\hline Compound/Sample & $\begin{array}{c}\mathbf{V} 4 \\
\boldsymbol{T}_{\mathbf{1} / 2} / \boldsymbol{K} \\
\left(\mathbf{d} / \mathbf{d}^{-\mathbf{1}}\right)\end{array}$ & $\begin{array}{c}\mathbf{V 8} \\
\boldsymbol{T}_{\mathbf{1} / 2} / \boldsymbol{K} \\
\left(\mathbf{d} / \mathbf{d}^{-\mathbf{1}}\right)\end{array}$ & $\begin{array}{c}\mathbf{V 1 6} \\
\boldsymbol{T}_{\mathbf{1} / 2} / \boldsymbol{K} \\
\left(\mathbf{d} / \mathbf{d}^{-\mathbf{1}}\right)\end{array}$ & $\begin{array}{c}\mathbf{E E} \\
\boldsymbol{T}_{1 / 2} / \boldsymbol{K} \\
\left(\mathbf{d} / \mathbf{d}^{-\mathbf{1}}\right)\end{array}$ & $\begin{array}{c}\mathbf{E E - S A} \\
\boldsymbol{T}_{\mathbf{1} / 2} / \boldsymbol{K} \\
\left(\mathbf{d} / \mathbf{d}^{-1}\right)\end{array}$ \\
\hline $\begin{array}{l}\text { Delphinidin 3-o- } \\
\text { glucoside }\end{array}$ & 35 & 36 & 29 & 41 & 41 \\
\hline $\begin{array}{l}\text { Cyanidin 3-o- } \\
\text { glucoside }\end{array}$ & 0.0195 & 0.0190 & 0.0233 & 0.0168 & 0.0168 \\
\hline $\begin{array}{l}\text { Petunidin 3-o- } \\
\text { glucoside }\end{array}$ & 42 & 55 & 52 & 33 & 42 \\
\hline $\begin{array}{l}\text { Malvidin 3-o- } \\
\text { glucoside }\end{array}$ & 0.0163 & 0.0126 & 0.0132 & 0.0204 & 0.0162 \\
\hline
\end{tabular}


A study conducted by Lavelli et al., (2017) in an ethanolic extract of grape pomace maltodextrin-encapsulated showed a low first-order rate constant, 0.0033-0.0014 $\mathrm{d}^{-1}$. This

344 might be due to lower water activity content and therefore these results are not comparable

345 with the present results, as this study assessed the stability of anthocyanins in a liquid form.

346 Moreover, the drying process will require higher overhead costs, and needs high energy and

347 pressure input which would add up greatly to the overall cost.

The half-lives of anthocyanins of EE and EE-SA stored at $20^{\circ} \mathrm{C}$ were 43 and $57 \mathrm{~d}$,

349 respectively (Table 1A). As discussed above, the degradation is faster in the CGA samples

350 with lowest surfactant concentration so V4 had the shortest half-life of $31 \mathrm{~d}$, followed by V8

351 (44 d) and V16 (55 d). Moreover the half-life of V16 was almost similar to EE-SA's, but

352 longer than that of EE. These half-life values were higher than the ones reported for the

353 blueberry juice stored at $25^{\circ} \mathrm{C}\left(\mathrm{t}_{1 / 2}=4.4 \mathrm{~d}\right)$, possibly due to the different types of

354 anthocyanins present (Buckow et al., 2010). Similarly, when the percentage of anthocyanin

355 losses after 32 days of storage was determined, the EE-SA had the least loss (31.53\%),

356 closely followed by V16 (34.90\%).

357 In summary, from all the above data it can be concluded that the surfactant had a

358 stabilisation effect on anthocyanins and this effect was comparable to that observed in

359 extracts with sorbic acid. To the best of our knowledge, only one report by Thakur \& Arya

360 (1989) assessed the stability of anthocyanins in grape juice preserved with sorbic acid and

361 their result agreed with the findings in this study. This further confirmed that the surfactant

362 might play an important role in protecting the anthocyanins from oxidation, thus extending

363 the half-life. 


\subsection{Anthocyanins Identification and Quantification}

The HPLC-DAD analysis showed that all samples had 13 anthocyanins identified at the beginning and at the end of storage study (Figure S1), which was in agreement with our previous study (MohdMaidin et al., 2017). The identified anthocyanins were: delphinidin, cyanidin, petunidin, peonidin and malvidin with different glycosyl acylation attached. In red wines and their pomace made from $V$. vinifera grapes, the main anthocyanins detected were of 3-o-monoglucosides of the free anthocyanidins including pelargonidin-3-o-glucoside, cyanidin-3-o-glucoside, delphinidin-3-o-glucoside, peonidin-3-o-glucoside, petunidin-3-oglucoside and malvidin-3-o-glucoside (Drosou et al., 2015; He et al., 2012; Kammerer et al., 2004). However, in this study, pelargonidin-3-o-glucoside was not detected and four anthocyanins (malvidin 3-o-glucoside, cyanidin 3-o-glucoside, delphinidin-3-o-glucoside, and petunidin-3-o-glucoside) were quantified as these are the most abundant anthocyanins present.

In all the samples, the most abundant anthocyanin was malvidin-3-o-glucoside $379(0.68 \mathrm{mg} / \mathrm{g})$ which was in agreement with other studies (Bimpilas, Panagopoulou, 380 Tsimogiannis, \& Oreopoulou, 2016; Morais, Ramos, Forgács, Cserháti, \& Oliviera, 2002) followed by delphinidin3-o-glucoside $(0.58 \mathrm{mg} / \mathrm{g})$. Both pigments were typically responsible

382 for the purple and purple-blue which could be seen in the colour of the ethanolic extract. except in EE. This can be seen in their short half-lives (29-41d). Fleschhut, Kratzer,

386 Rechkemmer, \& Kulling (2006) reported that an increase in hydroxyl groups in the B ring of the anthocyanin nucleus could result in a decrease in the stability which could possibly account for the anthocyanins loss. However, this was not observed in EE where cyanidin degraded faster than delphinidin, but both of them seemed to be less stable than petunidin 
390 and malvidin indicating that methylation of hydroxyl-groups in B ring increased the stability

391 of anthocyanins. Our results were comparable to those reported by Helllstrom et al., (2013)

392 for delphinidin and cyanidin in the blackcurrant and chokeberry juices stored at $21^{\circ} \mathrm{C}$ with

393 half-lives between 16-44 days.

Malvidins are known to be the most stable as compared to other anthocyanins due to

395 the absence of two hydroxyl groups in the B ring structure. This was clearly evident as they

396 had the longest half-life as compared to other anthocyanins across all samples. Interestingly,

397 malvidin in V16 sample had longer half-life (71d), with slowest degradation rate $(k=0.0097$

$\left.398 \mathrm{~d}^{-1}\right)$ than any of the control samples including EE-SA $\left(k=0.0116 \mathrm{~d}^{-1}, \mathrm{t}_{1 / 2}=65 \mathrm{~d}\right)$ which agrees

399 with the above observation on the protecting effect of the surfactant.

400 According to Hellström et al., (2013), the effect of the sugar moiety was minor as 401 compared to the effect induced by the type of the core anthocyanidin. In these extracts, 402 malvidin- and delphinidin 3-glucosides were the two predominant anthocyanins.

403 Delphinidin glucosides exhibited greater temperature sensitivity due to their three hydroxyl 404 group in the B ring in comparison to malvidin derivative which had only one $-\mathrm{OH}$ group 405 attached to it (Buckow et al., 2010). This can be clearly seen in the half-life of malvidin-3406 glucoside $\left(\mathrm{t}_{1 / 2}=55-71\right.$ days $)$ across all samples. Moreover, the stability of anthocyanins was 407 also reduced by the number of hydroxyl groups in the A ring with the absence of dihydroxyl 408 group in the B ring (Buckow et al., 2010). The matrix of samples also has been reported to 409 have a major impact on the stability of anthocyanins where anthocyanins in juices were more 410 prone to degradation as compared to those in smoothies, where the anthocyanins may be 411 protected by other phenolic compounds but the concrete reasons of this impact remained 412 unclear (Hellström et al., 2013). A study on anthocyanins stability from encapsulated grape 413 skin showed significant increase in the half-life of anthocyanins up to 452 days. The study 414 also proved that lowering the water activity of the encapsulated grape skin powder can 
415 double the half-life up to 998 days (Lavelli et al., 2017). In fruit juices, several factors can

416 influence the stability of anthocyanins, such as $\mathrm{pH}$, presence of ascorbic acid and

417 anthocyanin degrading enzymes (Buckow et al., 2010). Finally, the degree of glycosylation

418 also might possibly affect anthocyanins stability; the higher the degree of glycosylation, the

419 more stable they became.

420 Co-pigmentation of anthocyanins with other compounds is considered as an

421 important mechanism of colour stabilisation in plants. Anthocyanins can form co-pigments

422 with metal ions, other phenolic compounds or through self- association (Castañeda-Ovando,

423 Pacheco-Hernández, Páez-Hernández, Rodríguez, \& Galán-Vidal, 2009). Co-pigmentation

424 through self-association is unlikely because in order for it to take part, the concentration of

425 the anthocyanin should be greater than $1 \mathrm{mmol} / / \mathrm{L}$. Hydroxycinnamic acids and flavonols

426 were reported as the best cofactors in wine (Bimpilas et al., 2016). Co-pigmentation can be

427 influenced by the anthocyanins and co-pigment structure, and also by the concentration of

428 anthocyanins to the co-pigment (Eiro \& Heinonen, 2002). In the present study, the ratio of

429 anthocyanins and co-pigments might not be sufficient for the co-pigmentation to occur since

430 no additional phenolic acids were added to the samples. Thus, the stabilisation effect

431 observed in this study was solely due to the surfactant and the addition of sorbic acid.

433 3.5. Colour stability and $\mathrm{pH}$

434 Grape pomace extract had high levels of anthocyanins. However, anthocyanins 435 undergo degradation during processing and storage, thus affecting colour characteristics. In 436 the present study, the colour stability of the control samples and the CGA processed samples 437 was investigated and compared against EE and EE-SA. Colourimetric parameters chroma $438(C)$ and hue $(h)$ and $\Delta \mathrm{E}$ were determined to assess colour changes over time. The effects 439 were comparable to those observed in anthocyanins, yet with some exceptions. 
Figure 4 ( $\mathrm{A}$ and $\mathrm{B}$ ) showed the changes of colour in chroma and hue angle for the

441 control samples and the CGA processed samples over time. The results obtained showed that

442 EE and EE-SA had a similar trend; $\Delta \mathrm{E}$ values overlapped with each other. At day 0 , both

443 samples had dark red colour $(\mathrm{c}=39.4$, hue $=6.9)$. Over time, both chroma and hue values

444 decreased rapidly by day 22 , from dark red tending towards blue-black shade $(\mathrm{c}=14.9$, hue $445=-29.6)$.

The same trend was observed in all of the CGA processed samples, although the 447 chroma and hue angles decreased steadily as compared to EE and EE-SA. At day 0, all of 448 the CGA processed samples had almost similar colour of dark red shade $(c=49.2-51.5$; hue $449=8.7-15.1)$. However, the chroma $(\mathrm{c}=31.7-40.6)$ and hue angle (hue $=-1.4-2.8)$ values 450 decreased in all of the CGA processed samples over time. In short, V4 turned from dark red 451 to light red, tending towards blackish; V8 turned from dark red to light red, tending towards 452 browning and finally, V16 turned from dark red to light red, tending towards pinkish. 453 Therefore, these results showed that minimum colour changes were observed in V16, which 454 correlated with the lower degradation rate of anthocyanins determined above and confirms 455 the stabilisation effect by the surfactant.
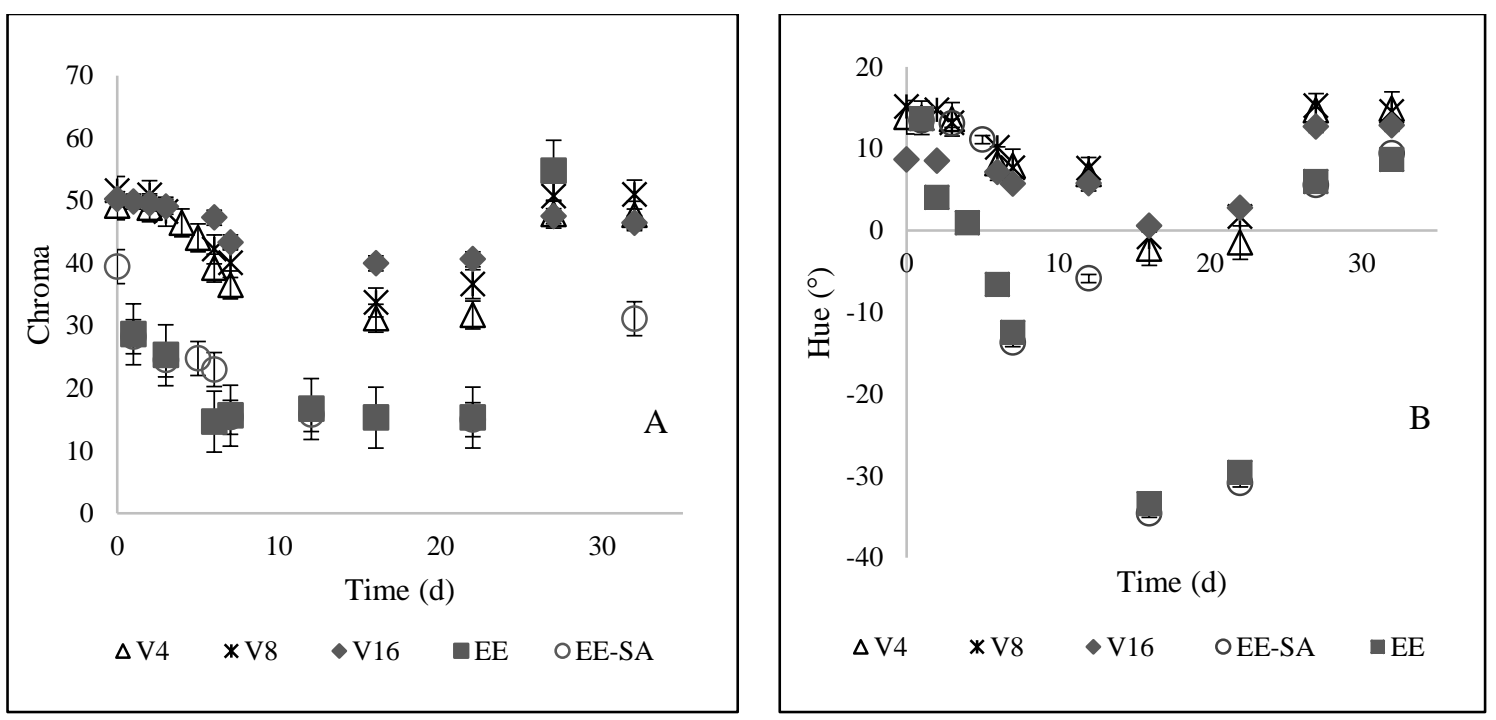


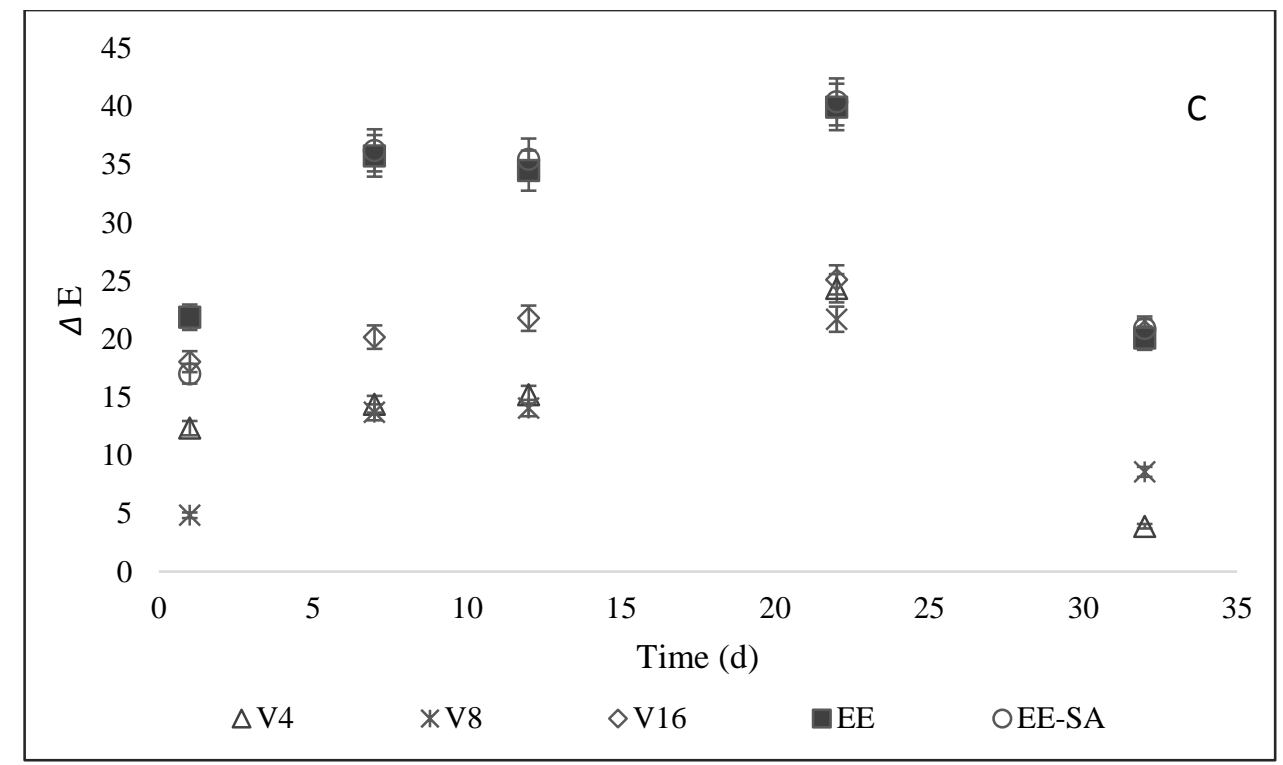

456 Figure 4. Chroma and hue values of samples during storage (A and B), total colour difference $(\Delta \mathrm{E})$ 457 between samples $(C)$. Error bars represent means \pm SD $(n=3)$. V4, CGA fraction separated at 458 volumetric ratio 4; V8, CGA fraction separated at volumetric ratio 8; V16, CGA fraction separated 459 at volumetric ratio 16; EE, crude ethanolic extract and EE-SA, ethanolic extract with sorbic acid.

Furthermore, the colour changes of samples can be further explained by $\Delta \mathrm{E}$ (Fig. 4C).

$462 \Delta \mathrm{E}$ indicates the magnitude of the colour difference between fresh and stored grape extracts

463 for different time points. Higher colour differences were measured for the control samples

464 than for the CGA samples. In all the CGA processed samples, the changes were not 465 significantly different $(\mathrm{p}>0.05)$ in the first 12 days of storage. However, higher magnitude 466 of changes was observed in day 22 , and minimal changes were observed in day 32.

Anthocyanins differ from each other by the number and position of the hydroxyl, and 468 methoxyl substituent groups in the B ring of the molecule. The hydroxylation pattern of the 469 anthocyanins in the B ring can directly affect the hue and colour stability due to the effect 470 on the delocalized electron path length in the molecule (He et al., 2012). Anthocyanins with 471 more hydroxyl groups in the B ring can contribute more to blueness, meanwhile the degree 472 of methylation in the B ring can increase redness. The rapid decrease in red colour of EE 473 and EE-SA might be explained by the degradation of a particular anthocyanin. In both 
474 control samples, cyanidin-3-glucoside and petunidin-3-glucoside had the shortest half-lives 475 between 16-21 days (Table 1B). Both anthocyanins were responsible for the red and dark

476 red colour respectively, which could explain the losses of dark red colour in both controlled

477 samples after 21 days. Both anthocyanins had two hydroxyl groups attached to the B ring,

478 which increased the blueness of the colour, as found in these samples. In the case of the CGA

479 processed samples, V4 appeared to have the same result as EE and EE-SA, which is 480 supported by the short half-life of cyanidin-3-glucoside determined in this sample. However, 481 in the case of V8 and V16, delpinidin-3-glucoside had the shortest half-life, 31 and 36 days 482 respectively. This could have contributed to the colour changes observed, from dark red to 483 light red, tending towards brownish and pinkish. Delphinidin-3-glucoside is responsible for 484 the blueness as it has three hydroxyl groups attached to the B ring.

Although most studies showed that delphinidin-3-glucoside exhibited a greater 486 thermal sensitivity due to their three hydroxyl substitution group, this was not clearly 487 observed in this study; thus the correlation between anthocyanin stability and chemical 488 structure is still unclear (Rice-Evans, Miller, \& Paganga, 1996). Moreover, the colour 489 changes in EE-SA could not be explained by the slowest anthocyanins loss in this sample. 490 This suggests that the mechanism of colour stabilisation in this sample needs further study 491 as colour change does not correlate with anthocyanins degradation. The mechanisms of 492 stabilisation of anthocyanins by TWEEN20 are yet to be determined, but we propose that 493 the micelles might play a role in encapsulating the anthocyanins protecting them against 494 oxidation during storage. 


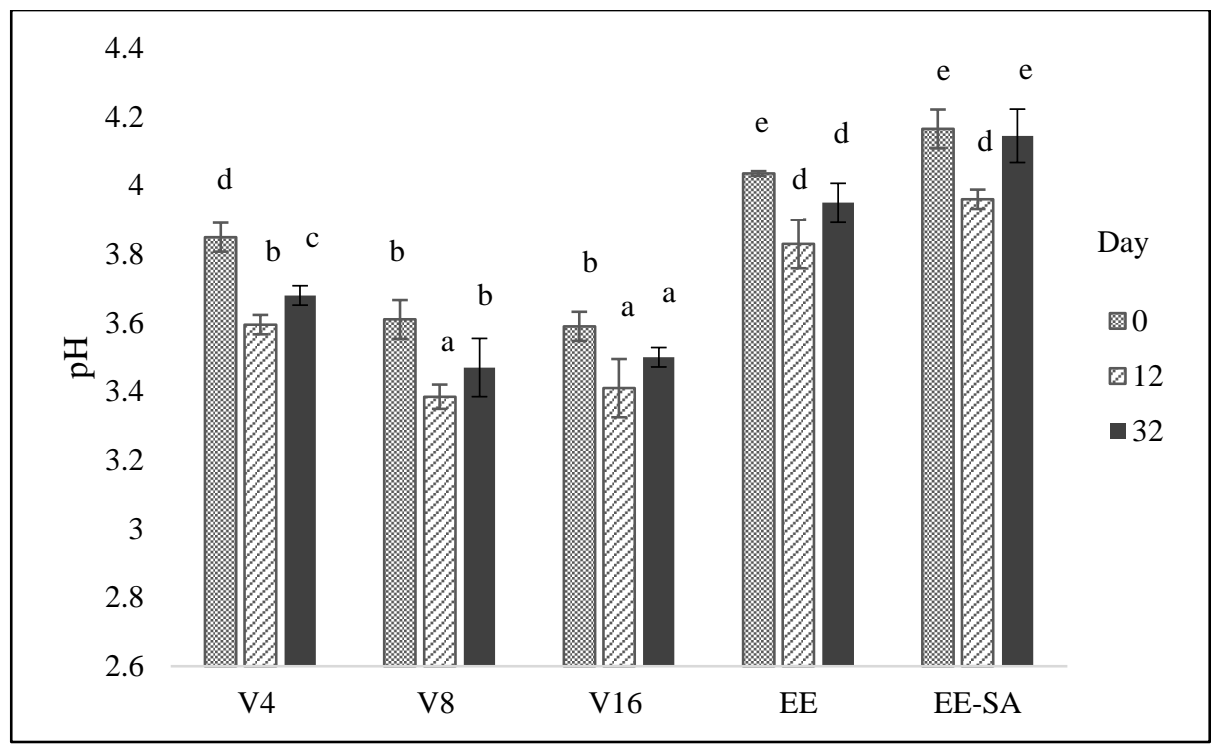

Figure 5. $\mathrm{pH}$ values of all samples on selected days. Error bars represent means $\pm \mathrm{SD}, \mathrm{n}=3$. V4, CGA fraction separated at volumetric ratio 4; V8, CGA fraction separated at volumetric ratio 8; V16, CGA fraction separated at volumetric ratio 16; EE, crude ethanolic extract and EE-SA, ethanolic extract with sorbic acid.

Overall, the $\mathrm{pH}$ of all samples dropped and then increased slightly (Fig. 5). Although

502 it is known that $\mathrm{pH}$ plays an important role in determining the state of the flavylium ion, the

503 trend was unclear in the present study. This could possibly be due to the presence of

504 TWEEN20 which could have a stabilisation effect as shown by the reduction of $\mathrm{pH}$ after

505 CGA separation. The increased in $\mathrm{pH}$ values later throughout the end of storage may be due

506 to the formation of phenolic acids like gallic acid; this can be supported by the fluctuations

507 in total phenolic content over storage which may be also an indication of the formation of

508 these intermediate compounds (Pérez-Jiménez, Neveu, Vos, \& Scalbert, 2010).

$510 \quad$ 4. Conclusions

511 The stability of the ethanolic raw extract from grape marc was compared with that of

512 the further processed sample after applying the CGA separation. The main effect was found 513 on the stability of anthocyanins. Anthocyanins stability in CGA fraction V16 was higher 
514 than in the raw extract based on the comparison of the first order kinetics of anthocyanins

515 degradation followed by all the samples. The stability in CGA samples increased with an

516 increase in surfactant concentration, V16 sample having the highest half-life (55 d) and

517 similar to the raw extract's with sorbic acid (57 d). Thus these results show that the surfactant

518 has a stabilization effect on the anthocyanins and the sorbic acid seems to have a similar

519 effect. Moreover a good correlation between the colour changes and degradation rate of

520 individual anthocyanins was observed whereby malvidin-3-o-glucoside was found to be the

521 most stable anthocyanin at all the studied conditions with the highest half-life found in V16.

522 Overall, this study shows that the surfactant has a stabilisation effect on the anthocyanins

523 and half-lives determined here were higher than others reported for wet formulations of

524 anthocyanins. The mechanism of stabilisation of anthocyanins by TWEEN20 may be related

525 to the solubilisation of the anthocyanins within the micelles. Furthermore, the main findings

526 in this study have shown the advantages of CGA as a separation method that can also

527 integrate a pre-formulation step.

529 Acknowledgement

530 The authors would like to thank the Ministry of Higher Education Malaysia (MOHE) and

531 Universiti Malaysia Terengganu (UMT) for their financial support. The authors are also

532 grateful to winery in Northern Italy for providing the grape pomace.

\section{Conflict of interest}

535 The authors declare no conflict of interest. 
539

Ainsworth, E. A., \& Gillespie, K. M. (2007). Estimation of total phenolic content and other oxidation substrates in plant tissues using Folin-Ciocalteu reagent. Nature Protocols, 2(4), 875-877. https://doi.org/10.1038/nprot.2007.102

Amendola, D., De Faveri, D. M., \& Spigno, G. (2010). Grape marc phenolics: Extraction kinetics, quality and stability of extracts. Journal of Food Engineering, 97, 384-392. https://doi.org/10.1016/j.jfoodeng.2009.10.033

Barba, F. J., Zhu, Z., Koubaa, M., Sant'Ana, A. S., \& Orlien, V. (2016). Green alternative methods for the extraction of antioxidant bioactive compounds from winery wastes and by-products: A review. Trends in Food Science and Technology, 49, 96-109. https://doi.org/10.1016/j.tifs.2016.01.006

Bimpilas, A., Panagopoulou, M., Tsimogiannis, D., \& Oreopoulou, V. (2016). Anthocyanin copigmentation and color of wine: The effect of naturally obtained hydroxycinnamic acids as cofactors. Food Chemistry, 197, 39-46. https://doi.org/10.1016/j.foodchem.2015.10.095

Buckow, R., Kastell, A., Terefe, N. S., \& Versteeg, C. (2010). Pressure and temperature effects on degradation kinetics and storage stability of total anthocyanins in blueberry juice. Journal of Agricultural and Food Chemistry, 58(18), 10076-10084. https://doi.org/10.1021/jf1015347

Cardona, J. A., Lee And, J. H., \& Talcott, S. T. (2009). Color and polyphenols stability in extracts produced from muscadine grape (vitis rotundifolia) pomace. Journal of Agricultural and Food Chemistry, 57(18), 8421-8425. https://doi.org/10.1021/jf901840t

Castañeda-Ovando, A., Pacheco-Hernández, M. de L., Páez-Hernández, M. E., Rodríguez, J. A., \& Galán-Vidal, C. A. (2009). Chemical studies of anthocyanins: A review. Food Chemistry, 113(4), 859-871. https://doi.org/10.1016/j.foodchem.2008.09.001

Clemente, E., \& Galli, D. (2011). Stability of the anthocyanins extracted from residues of the wine industry. Ciência E Tecnologia de Alimentos, 31(3), 765-768. https://doi.org/10.1590/S0101-20612011000300033

Clemente, E., \& Galli, D. (2013). Stability evaluation of anthocyanin extracted from processed grape residues. International Journal of Sciences, 2(July), 12-18.

Dermiki, M., Bourquin, A. L., \& Jauregi, P. (2010). Separation of astaxanthin from cells of Phaffia rhodozyma using colloidal gas aphrons in a flotation column. Biotechnology Progress, 26(2), 477-487. https://doi.org/10.1002/btpr.340

Dermiki, M., Gordon, M. H., \& Jauregi, P. (2009). Recovery of astaxanthin using colloidal gas aphrons (CGA): A mechanistic study. Separation and Purification Technology, 65(1), 54-64. https://doi.org/10.1016/j.seppur.2007.12.023

Drosou, C., Kyriakopoulou, K., Bimpilas, A., Tsimogiannis, D., \& Krokida, M. (2015). A comparative study on different extraction techniques to recover red grape pomace polyphenols from vinification byproducts. Industrial Crops and Products, 75, 141149. https://doi.org/10.1016/j.indcrop.2015.05.063 
Eiro, M. J., \& Heinonen, M. (2002). Anthocyanin color behavior and stability during storage: Effect of intermolecular copigmentation. Journal of Agricultural and Food Chemistry, 50(25), 7461-7466. https://doi.org/10.1021/jf0258306

Falguera, V., Quintero, J. P., Jiménez, A., Muñoz, J. A., \& Ibarz, A. (2011). Edible films and coatings: Structures, active functions and trends in their use. Trends in Food Science and Technology, 22(6), 292-303. https://doi.org/10.1016/j.tifs.2011.02.004

Fleschhut, J., Kratzer, F., Rechkemmer, G., \& Kulling, S. E. (2006). Stability and biotransformation of various dietary anthocyanins in vitro. European Journal of Nutrition, 45(1), 7-18. https://doi.org/10.1007/s00394-005-0557-8

Fontana, A. R., Antoniolli, A., \& Bottini, R. (2013). Grape pomace as a sustainable source of bioactive compounds: Extraction, characterization, and biotechnological applications of phenolics. Journal of Agricultural and Food Chemistry, 61(38), 89879003. https://doi.org/10.1021/jf402586f

Fuda, E., Bhatia, D., Pyle, D. L., \& Jauregi, P. (2005). Selective separation of $\beta$ lactoglobulin from sweet whey using CGAs generated from the cationic surfactant CTAB. Biotechnology and Bioengineering, 90(5), 532-542. https://doi.org/10.1002/bit.20412

Fuda, E., \& Jauregi, P. (2006). An insight into the mechanism of protein separation by colloidal gas aphrons (CGA) generated from ionic surfactants. Journal of Chromatography B: Analytical Technologies in the Biomedical and Life Sciences, 843(2), 317-326. https://doi.org/10.1016/j.jchromb.2006.06.032

He, F., Liang, N. N., Mu, L., Pan, Q. H., Wang, J., Reeves, M. J., \& Duan, C. Q. (2012). Anthocyanins and their variation in red wines I. Monomeric anthocyanins and their color expression. Molecules, 17(2), 1571-1601. https://doi.org/10.3390/molecules17021571

Hellström, J., Mattila, P., \& Karjalainen, R. (2013). Stability of anthocyanins in berry juices stored at different temperatures. Journal of Food Composition and Analysis, 31(1), 12-19. https://doi.org/10.1016/j.jfca.2013.02.010

Kammerer, D., Claus, A., Carle, R., \& Schieber, A. (2004). Polyphenol Screening of Pomace from Red and White Grape Varieties (Vitis vinifera L.) by HPLC-DADMS/MS. Journal of Agricultural and Food Chemistry, 52, 4360-4367.

Kammerer, D. R., Kammerer, J., Valet, R., \& Carle, R. (2014). Recovery of polyphenols from the by-products of plant food processing and application as valuable food ingredients. Food Research International, 65(PA), 2-12.

https://doi.org/10.1016/j.foodres.2014.06.012

Lavelli, V., Harsha, P. S. C. S., Laureati, M., \& Pagliarini, E. (2017). Degradation kinetics of encapsulated grape skin phenolics and micronized grape skins in various water activity environments and criteria to develop wide-ranging and tailor-made food applications. Innovative Food Science \& Emerging Technologies, 39, 156-164. https://doi.org/http://dx.doi.org/10.1016/j.ifset.2016.12.006

Lee, J., Rennaker, C., \& Wrolstad, R. E. (2008). Correlation of two anthocyanin quantification methods: HPLC and spectrophotometric methods. Food Chemistry, 
Lin, Q., Wang, J., Qin, D., \& Bergenståhl, B. (2007). Influence of amphiphilic structures on the stability of polyphenols with different hydrophobicity. Science in China, Series B: Chemistry, 50(1), 121-126. https://doi.org/10.1007/s11426-007-0009-9

Lozovskaya, T., Brenner Weiss, G., Franzreb, M., \& Nusser, M. (2012). Recovery of Anthocyanins From Grape Pomace Extract (Pinot Noir) Using Magnetic Particles Based on Poly(Vinyl Alcohol). Cellulose Chemistry and Technology, 46(7-8), 427433.

MohdMaidin, N., Michael, N., Oruna-Concha, M. J., \& Jauregi, P. (2017). Polyphenols extracted from red grape pomace by a surfactant based method show enhanced collagenase and elastase inhibitory activity. Journal of Chemical Technology and Biotechnology. https://doi.org/10.1002/jctb.5459

Morais, H., Ramos, C., Forgács, E., Cserháti, T., \& Oliviera, J. (2002). Influence of storage conditions on the stability of monomeric anthocyanins studied by reversed-phase high-performance liquid chromatography. Journal of Chromatography B: Analytical Technologies in the Biomedical and Life Sciences, 770(1-2), 297-301. https://doi.org/10.1016/S1570-0232(02)00055-7

Pérez-Jiménez, J., Neveu, V., Vos, F., \& Scalbert, A. (2010). Identification of the 100 richest dietary sources of polyphenols: an application of the Phenol-Explorer database. European Journal of Clinical Nutrition, 64, S112-S120. https://doi.org/10.1038/ejcn.2010.221

Presilski, S., Presilska, N., \& Tomovska, D. (2016). Effects of Extraction, Conventional Processing and Storage on Natural Anthocyanins. Journal of Food Processing \& Technology, 7(2), 2-4. https://doi.org/10.4172/2157-7110.1000551

Quirós-Sauceda, A. E., Ayala-Zavala, J. F., Olivas, G. I., \& González-Aguilar, G. A. (2014). Edible coatings as encapsulating matrices for bioactive compounds: a review. Journal of Food Science and Technology, 51(9), 1674-1685. https://doi.org/10.1007/s13197-013-1246-X

Re, R., Pellegrini, N., Proteggente, A., Pannala, A., Yang, M., \& Rice-Evans, C. (1999). Antioxidant activity applying an improved ABTS radical cation decolorization assay. Free Radical Biology and Medicine, 26(9-10), 1231-1237. https://doi.org/10.1016/S0891-5849(98)00315-3

Rice-Evans, C., Miller, N., \& Paganga, G. (1996). Structure-antioxidant activity relatioships of flavonoids and phenolic acids. Free Radical Biology and Medicine, 20(7), 933-956.

Singleton, V. L., \& Rossi, J. A. J. (1965). Colorimetry of total phenolics with acid reagents. Am J Enol Vitic, 16, 144-158.

Spigno, G., Amendola, D., Dahmoune, F., \& Jauregi, P. (2015). Colloidal gas aphrons based separation process for the purification and fractionation of natural phenolic extracts. Food and Bioproducts Processing, 94, 434-442. https://doi.org/10.1016/j.fbp.2014.06.002 
Spigno, G., Dermiki, M., Pastori, C., Casanova, F., \& Jauregi, P. (2010). Recovery of gallic acid with colloidal gas aphrons generated from a cationic surfactant. Separation and Purification Technology, 71(1), 56-62. https://doi.org/10.1016/j.seppur.2009.11.002

Thakur, B. R., \& Arya, S. S. (1989). Studies on stability of blue grape anthocyanins.

668 International Journal of Food Science \& Technology, 24(3), 321-326.

669 https://doi.org/10.1111/j.1365-2621.1989.tb00650.x

Troller, J. A., \& Olsen, R. A. (1967). Derivatives of Sorbic Acid as Food Preservatives. Journal of Food Science, 32, 228-231.

Yu, J., \& Ahmedna, M. (2013). Functional components of grape pomace: Their composition, biological properties and potential applications. International Journal of Food Science and Technology, 48(2), 221-237. https://doi.org/10.1111/j.1365- 